\title{
Analysis, adaptive control and synchronization of a novel 4-D hyperchaotic hyperjerk system via backstepping control method
}

\author{
SUNDARAPANDIAN VAIDYANATHAN
}

\begin{abstract}
A hyperjerk system is a dynamical system, which is modelled by an $n$th order ordinary differential equation with $n \geqslant 4$ describing the time evolution of a single scalar variable. Equivalently, using a chain of integrators, a hyperjerk system can be modelled as a system of $n$ first order ordinary differential equations with $n \geqslant 4$. In this research work, a 4-D novel hyperchaotic hyperjerk system with two nonlinearities has been proposed, and its qualitative properties have been detailed. The novel hyperjerk system has a unique equilibrium at the origin, which is a saddle-focus and unstable. The Lyapunov exponents of the novel hyperjerk system are obtained as $L_{1}=0.14219, L_{2}=0.04605, L_{3}=0$ and $L_{4}=-1.39267$. The Kaplan-Yorke dimension of the novel hyperjerk system is obtained as $D_{K Y}=3.1348$. Next, an adaptive controller is designed via backstepping control method to stabilize the novel hyperjerk chaotic system with three unknown parameters. Moreover, an adaptive controller is designed via backstepping control method to achieve global synchronization of the identical novel hyperjerk systems with three unknown parameters. MATLAB simulations are shown to illustrate all the main results derived in this research work on a novel hyperjerk system.
\end{abstract}

Key words: hyperchaos, hyperjerk system, adaptive control, backstepping control, synchronization.

\section{Introduction}

Chaos theory describes the qualitative study of unstable aperiodic behaviour in deterministic nonlinear dynamical systems. For the motion of a dynamical system to be chaotic, the system variables should contain nonlinear terms and it must satisfy three properties: boundedness, infinite recurrence and sensitive dependence on initial conditions $[1,2]$.

The first famous chaotic system was accidentally discovered by Lorenz, when he was designing a 3-D model for atmospheric convection in 1963 [3]. Subsequently, Rössler discovered a 3-D chaotic system in 1976 [4], which is algebraically simpler than the Lorenz system.

The Author is with Research and Development Centre, Vel Tech University, Avadi, Chennai- 600062, Tamilnadu, India. E-mail: sundarvtu@gmail.com.

Received 9.03.2016. 
Some well-known paradigms of 3-D chaotic systems are Arneodo system [5], Sprott systems [6], Chen system [7], Lü-Chen system [8], Liu system [9], Cai system [10], Tsystem [11], etc. Many new chaotic systems have been also discovered like Li system [12], Sundarapandian systems [13, 14], Vaidyanathan systems [15, 16, 17, 18, 19, 20, 21, 22, 23, 24, 25, 26, 27, 28, 29, 30, 31 , 32, 33, 34], Pehlivan system [35], Jafari system [36], Pham systems [37, 38, 39, 40], Sampath system [41], Akgul system [42], etc.

A hyperchaotic system is generally defined as a chaotic system with at least two positive Lyapunov exponents [43]. Thus, the dynamics of a hyperchaotic system are expended in several different directions simultaneously. Thus, the hyperchaotic systems have more complex dynamical behaviour and hence they have miscellaneous applications in engineering such as secure communications [44, 45], cryptosystems [46, 47], encryption [48, 49], electrical circuits [50, 51], etc.

The minimum dimension for an autonomous, continuous-time, hyperchaotic system is four. Since the discovery of a first 4-D hyperchaotic system by Rössler in 1979 [52], many 4-D hyperchaotic systems have been found in the literature such as hyperchaotic Lorenz system [53], hyperchaotic Lü system [54], hyperchaotic Chen system [55], hyperchaotic Wang system [56], hyperchaotic Newton-Leipnik system [57], hyperchaotic Jia system [58], hyperchaotic Vaidyanathan systems [59, 60, 61, 62, 63, 64, 65, 66, 67, 68], hyperchaotic Pham system [69], hyperchaotic Sampath system [70], etc.

In this paper, we propose a 4-D novel hyperchaotic hyperjerk system by adding a hyperbolic sinusoidal nonlinearity to the Chlouverakis-Sprott hyperjerk system [71].

First, we detail the fundamental qualitative properties of the novel hyperchaotic hyperjerk system. We show that the Lyapunov exponents of the novel hyperjerk system are given by $L_{1}=0.14219, L_{2}=0.04605, L_{3}=0$ and $L_{4}=-1.39267$. Since the sum of the Lyapunov exponents is negative, we deduce that the novel hyperjerk system is dissipative. Also, we show that the Kaplan-Yorke dimension of the novel hyperjerk system is obtained as $D_{K Y}=3.1348$.

The study of control of a chaotic system investigates methods for designing feedback control laws that globally or locally asymptotically stabilize or regulate the outputs of a chaotic system.

Next, this paper derives an adaptive backstepping control law that stabilizes the novel hyperjerk system, when the system parameters are unknown. The backstepping control method is a recursive procedure that links the choice of a Lyapunov function with the design of a controller and guarantees global asymptotic stability of strict feedback systems $[72,73,74,75]$.

This paper also derives an adaptive backstepping control law that achieves global chaos synchronization of the identical 4-D novel hyperchaotic hyperjerk systems with unknown parameters.

Chaos synchronization problem deals with the synchronization of a couple of systems called the master or drive system and the slave or response system. To solve this problem, control laws are designed so that the output of the slave system tracks the output of the master system asymptotically with time. 
In the chaos literature, an impressive variety of techniques have been proposed to solve the problem of chaos synchronization such as active control method [76, 77], adaptive control method [78, 79], backstepping control method [80, 81, 82, 83], sliding mode control method [84, 85, 86, 87, 88, 89], etc.

All the main adaptive results in this paper are derived using backstepping control method and proved using Lyapunov stability theory [90]. MATLAB simulations are depicted to illustrate the phase portraits of the novel hyperchaotic hyperjerk system with two positive Lyapunov exponents, adaptive stabilization and synchronization results for the novel 4-D hyperchaotic hyperjerk system.

\section{A 4-D novel hyperchaotic hyperjerk system}

In mechanics, a jerk system is described by an explicit third order ordinary differential equation describing the time evolution of a single scalar variable $x$ according to the dynamics

$$
\frac{d^{3} x}{d t^{3}}=f\left(\frac{d^{2} x}{d t^{2}}, \frac{d x}{d t}, x\right)
$$

The differential equation(1) is called a jerk system because the successive derivatives of the displacement in a mechanical system are the velocity, acceleration, and jerk.

A generalization of the jerk dynamics is given by the dynamics

$$
\frac{d^{(n)} x}{d t^{n}}=f\left(\frac{d^{(n-1)} x}{d t^{n-1}}, \ldots, \frac{d x}{d t}, x\right), \quad(n \geqslant 4)
$$

An ordinary differential equation of the form (2) is called a hyperjerk system since it involves time derivatives of a jerk function.

In [71], Chlouverakis and Sprott discovered a simple hyperchaotic hyperjerk system given by the dynamics

$$
\frac{d^{4} x}{d t^{4}}+\frac{d^{3} x}{d t^{3}} x^{4}+A \frac{d^{2} x}{d t^{2}}+\frac{d x}{d t}+x=0
$$

In system form, the differential equation (3) can be expressed as

$$
\left\{\begin{array}{l}
\dot{x}_{1}=x_{2} \\
\dot{x}_{2}=x_{3} \\
\dot{x}_{3}=x_{4} \\
\dot{x}_{4}=-x_{1}-x_{2}-A x_{3}-x_{1}^{4} x_{4}
\end{array}\right.
$$

When $A=3.6$, the hyperjerk system (4) exhibits hyperchaos with Lyapunov exponent spectrum $(0.132,0.035,0,-1.25)$. Thus, the maximum Lyapunov exponent (MLE) 
of the Chlouverakis-Sprott hyperchaotic hyperjerk system (4) is $L_{1}=0.132$ and the Kaplan-Yorke dimension of this hyperjerk system is easily calculated a $D_{K Y}=3.13$.

In this work, we propose a novel hyperjerk system by adding a hyperbolic sinusoidal nonlinearity to the Chlouverakis-Sprott hyperjerk system (4) and with a different set of values for the system parameters.

Our novel hyperjerk system is given in system form as

$$
\left\{\begin{array}{l}
\dot{x}_{1}=x_{2} \\
\dot{x}_{2}=x_{3} \\
\dot{x}_{3}=x_{4} \\
\dot{x}_{4}=-x_{1}-x_{2}-a x_{3}-b \sinh \left(x_{2}\right)-c x_{1}^{4} x_{4}
\end{array}\right.
$$

where $a, b$ and $c$ are positive parameters.

In this paper, we shall show that the system (5) is hyperchaotic when the parameters $a, b$ and $c$ take the values

$$
a=3.7, \quad b=0.05, \quad c=1.3
$$

For the parameter values in (6), the Lyapunov exponents of the novel hyperjerk system (5) are obtained as

$$
L_{1}=0.14219, L_{2}=0.04605, L_{3}=0, L_{4}=-1.39267
$$

From the LE spectrum given in (7), it is easily seen that the maximal Lyapunov exponent (MLE) of our novel hyperchaotic hyperjerk system (5) is $L_{1}=0.14219$, which is greater than the MLE of the Chlouverakis-Sprott hyperchaotic hyperjerk system (4). Also, the Kaplan-Yorke dimension of the novel hyperjerk system (5) is calculated as $D_{K Y}=3.1348$, which is greater than the Kaplan-Yorke dimension of the ChlouverakisSprott hyperjerk system (4). This shows that the novel hyperchaotic hyperjerk system (5) exhibits more complex behaviour than the Chlouverakis-Sprott hyperchaotic hyperjerk system (4).

For numerical simulations, we take the initial values of the novel hyperjerk system (5) as $x_{1}(0)=0.5, x_{2}(0)=0.5, x_{3}(0)=0.5$ and $x_{4}(0)=0.5$.

Figs. 1-4 depict the 3-D projections of the 4-D novel hyperjerk system (5) on $\left(x_{1}, x_{2}, x_{3}\right),\left(x_{1}, x_{2}, x_{4}\right),\left(x_{1}, x_{3}, x_{4}\right)$ and $\left(x_{2}, x_{3}, x_{4}\right)$ spaces respectively. 


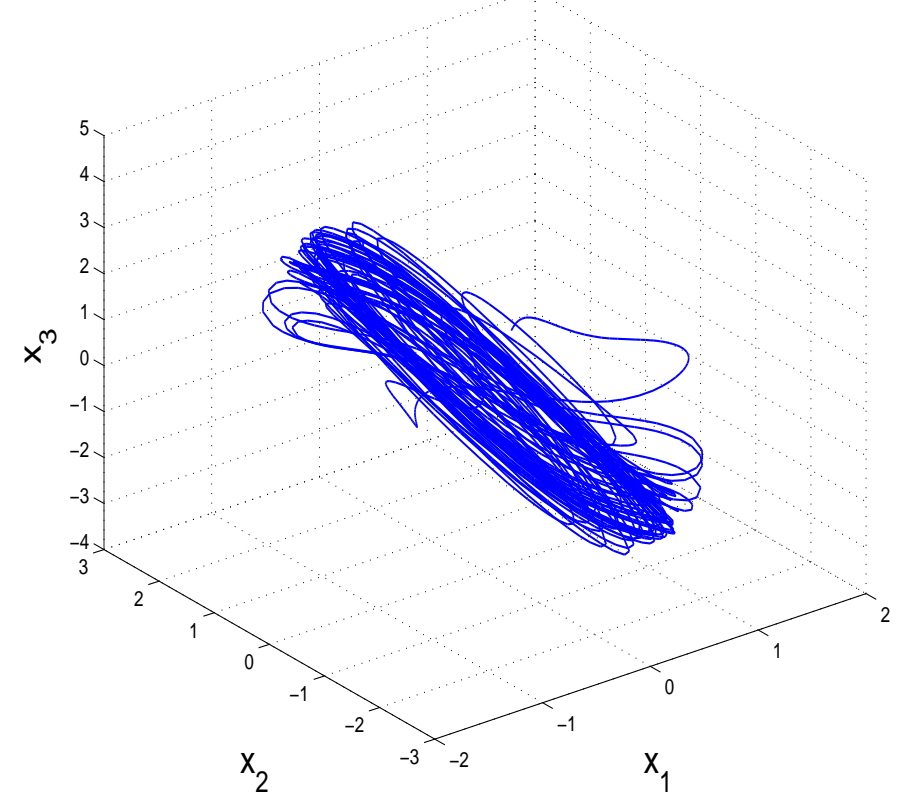

Figure 1: 3-D projection of the 4-D novel hyperjerk system on $\left(x_{1}, x_{2}, x_{3}\right)$ space

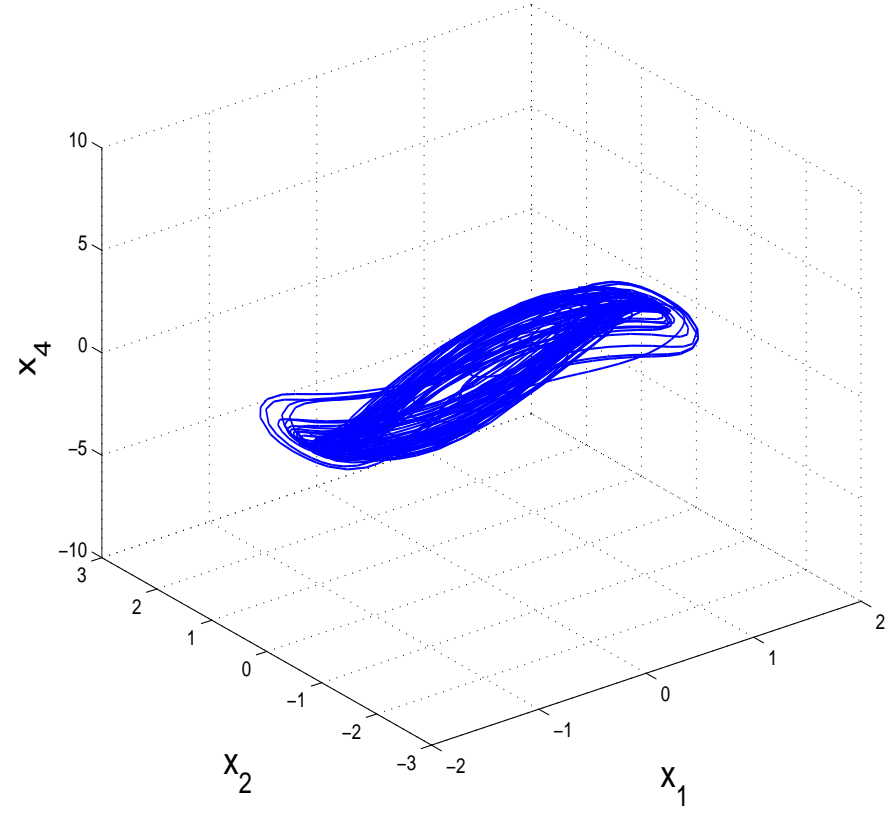

Figure 2: 3-D projection of the 4-D novel hyperjerk system on $\left(x_{1}, x_{2}, x_{4}\right)$ space 


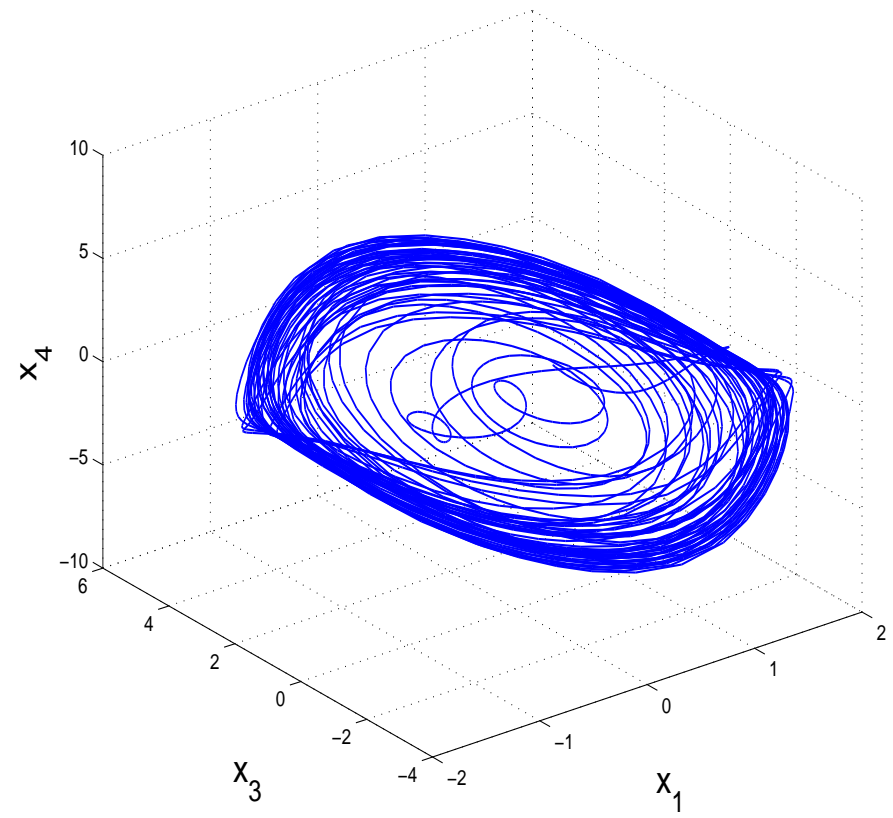

Figure 3: 3-D projection of the 4-D novel hyperjerk system on $\left(x_{1}, x_{3}, x_{4}\right)$ space

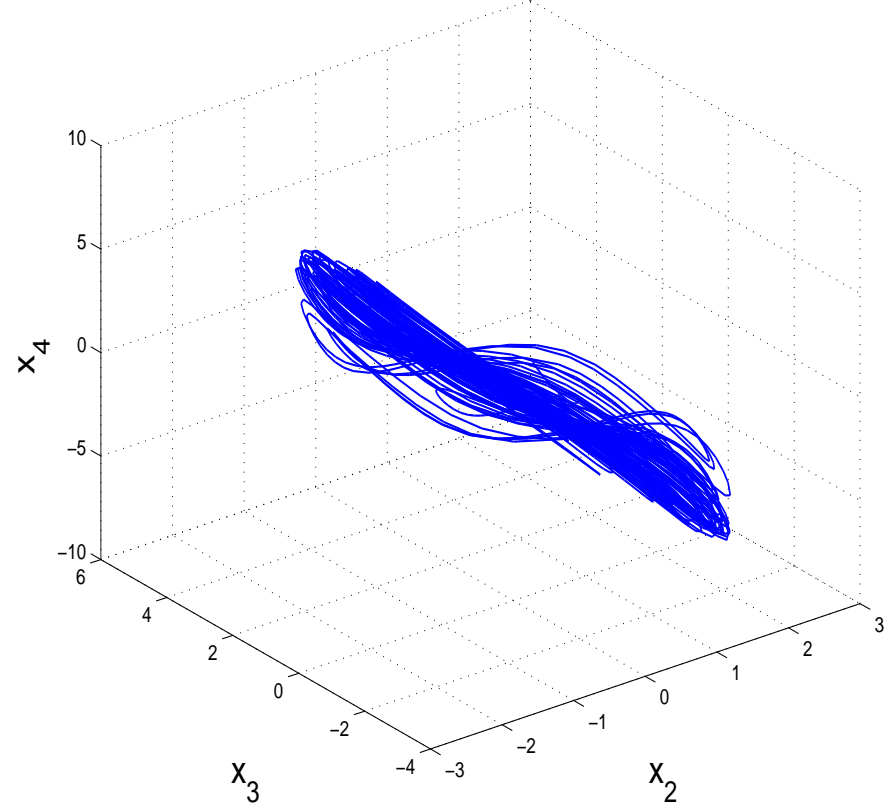

Figure 4: 3-D projection of the 4-D novel hyperjerk system on $\left(x_{2}, x_{3}, x_{4}\right)$ space 


\section{Analysis of the 4-D novel hyperjerk system}

\subsection{Equilibrium points}

The equilibrium points of the 4-D novel hyperjerk system (5) are obtained by solving the equations

$$
\left.\begin{array}{rlrl}
f_{1}\left(x_{1}, x_{2}, x_{3}, x_{4}\right) & =x_{2} & = & 0 \\
f_{2}\left(x_{1}, x_{2}, x_{3}, x_{4}\right) & =x_{3} & = & 0 \\
f_{3}\left(x_{1}, x_{2}, x_{3}, x_{4}\right) & =x_{4} & = & 0 \\
f_{4}\left(x_{1}, x_{2}, x_{3}, x_{4}\right) & =-x_{1}-x_{2}-a x_{3}-b \sinh \left(x_{2}\right)-c x_{1}^{4} x_{4} & = & 0
\end{array}\right\}
$$

We take the parameter values as in the hyperchaotic case (6).

Thus, the equilibrium points of the system (5) are characterized by the equations

$$
x_{1}=0, x_{2}=0, x_{3}=0, x_{4}=0
$$

Solving the system (9), we note that the 4-D novel hyperjerk system (5) has a unique equilibrium at the origin, i.e.

$$
E_{0}=\left[\begin{array}{l}
0 \\
0 \\
0 \\
0
\end{array}\right]
$$

To test the stability type of the equilibrium points $E_{0}$, we calculate the Jacobian matrix of the novel hyperjerk system (5) at $E_{0}$ as

$$
J_{0}=J\left(E_{0}\right)=\left[\begin{array}{cccc}
0 & 1 & 0 & 0 \\
0 & 0 & 1 & 0 \\
0 & 0 & 0 & 1 \\
-1 & -1-b & -a & 0
\end{array}\right]=\left[\begin{array}{cccc}
0 & 1 & 0 & 0 \\
0 & 0 & 1 & 0 \\
0 & 0 & 0 & 1 \\
-1 & -1.05 & -3.7 & 0
\end{array}\right]
$$

The matrix $J_{0}$ has the eigenvalues

$$
\lambda_{1,2}=0.1622 \pm 1.8695 i, \quad \lambda_{3,4}=-0.1662 \pm 0.5076 i
$$

This shows that the equilibrium point $E_{0}$ is a saddle-focus point, which is unstable. 


\subsection{Lyapunov exponents and Kaplan-Yorke dimension}

For the parameter values $a=3.7, b=0.05$ and $c=1.3$, the Lyapunov exponents of the novel hyperjerk system (5) are numerically obtained using MATLAB as

$$
L_{1}=0.14219, L_{2}=0.04605, L_{3}=0 \text { and } L_{4}=-1.39627
$$

Since the LE spectrum in (13) has two positive Lyapunov exponents, the novel hyperjerk system (5) is hyperchaotic.

Since $L_{1}+L_{2}+L_{3}+L_{4}=-1.2080<0$, it follows that the novel hyperjerk system (5) is dissipative.

Also, the Kaplan-Yorke dimension of the novel hyperchaotic hyperjerk system (5)is obtained as

$$
D_{K Y}=3+\frac{L_{1}+L_{2}+L_{3}}{\left|L_{4}\right|}=3.1348
$$

which is fractional.

\section{Adaptive control of the 4-D novel hyperjerk system with unknown parameters}

In this section, we use backstepping control method to derive an adaptive feedback control law for globally stabilizing the 4-D novel hyperjerk system with unknown parameters.

Thus, we consider the 4-D novel jerk chaotic system given by

$$
\left\{\begin{array}{l}
\dot{x}_{1}=x_{2} \\
\dot{x}_{2}=x_{3} \\
\dot{x}_{3}=x_{4} \\
\dot{x}_{4}=-x_{1}-x_{2}-a x_{3}-b \sinh \left(x_{2}\right)-c x_{1}^{4} x_{4}+u
\end{array}\right.
$$

where $a, b$ and $c$ are unknown constant parameters, and $u$ is a backstepping control law to be determined using estimates $\hat{a}(t), \hat{b}(t)$ and $\hat{c}(t)$ for $a, b$ and $c$, respectively.

The parameter estimation errors are defined as:

$$
\left\{\begin{array}{l}
e_{a}(t)=a-\hat{a}(t) \\
e_{b}(t)=b-\hat{b}(t) \\
e_{c}(t)=c-\hat{c}(t)
\end{array}\right.
$$


Differentiating (16) with respect to $t$, we obtain the following equations:

$$
\left\{\begin{array}{l}
\dot{e}_{a}(t)=-\dot{\hat{a}}(t) \\
\dot{e}_{b}(t)=-\dot{\hat{b}}(t) \\
\dot{e}_{c}(t)=-\dot{\hat{c}}(t)
\end{array}\right.
$$

Next, we shall state and prove the main result of this section.

Theorem 1 The 4-D novel hyperjerk system (15), with unknown parameters $a, b$ and $c$, is globally and exponentially stabilized by the adaptive feedback control law,

$$
u(t)=-4 x_{1}-9 x_{2}-[9-\hat{a}(t)] x_{3}-4 x_{4}+\hat{b}(t) \sinh \left(x_{2}\right)+\hat{c}(t) x_{1}^{4} x_{4}-k z_{4},
$$

where $k>0$ is a gain constant,

$$
z_{4}=3 x_{1}+5 x_{2}+3 x_{3}+x_{4}
$$

and the update law for the parameter estimates $\hat{a}(t), \hat{b}(t), \hat{c}(t)$ is given by

$$
\left\{\begin{array}{l}
\dot{\hat{a}}(t)=-x_{3} z_{4} \\
\dot{\hat{b}}(t)=-\sinh \left(x_{2}\right) z_{4} \\
\dot{\hat{c}}(t)=-x_{1}^{4} x_{4} z_{4}
\end{array}\right.
$$

Proof We prove this result via backstepping control and Lyapunov stability theory [90].

First, we define a quadratic Lyapunov function

$$
V_{1}\left(z_{1}\right)=\frac{1}{2} z_{1}^{2}
$$

where

$$
z_{1}=x_{1}
$$

Differentiating $V_{1}$ along the dynamics (15), we get

$$
\dot{V}_{1}=z_{1} \dot{z}_{1}=x_{1} x_{2}=-z_{1}^{2}+z_{1}\left(x_{1}+x_{2}\right)
$$

Now, we define

$$
z_{2}=x_{1}+x_{2}
$$

Using (24), we can simplify the equation (23) as

$$
\dot{V}_{1}=-z_{1}^{2}+z_{1} z_{2}
$$


Secondly, we define a quadratic Lyapunov function

$$
V_{2}\left(z_{1}, z_{2}\right)=V_{1}\left(z_{1}\right)+\frac{1}{2} z_{2}^{2}=\frac{1}{2}\left(z_{1}^{2}+z_{2}^{2}\right)
$$

Differentiating $V_{2}$ along the dynamics (15), we get

$$
\dot{V}_{2}=-z_{1}^{2}-z_{2}^{2}+z_{2}\left(2 x_{1}+2 x_{2}+x_{3}\right)
$$

Now, we define

$$
z_{3}=2 x_{1}+2 x_{2}+x_{3}
$$

Using (28), we can simplify the equation (27) as

$$
\dot{V}_{2}=-z_{1}^{2}-z_{2}^{2}+z_{2} z_{3}
$$

Thirdly, we define a quadratic Lyapunov function

$$
V_{3}\left(z_{1}, z_{2}, x_{3}\right)=V_{2}\left(z_{1}, z_{2}\right)+\frac{1}{2} z_{3}^{2}=\frac{1}{2}\left(z_{1}^{2}+z_{2}^{2}+z_{3}^{2}\right)
$$

Differentiating $V_{3}$ along the dynamics (15), we get

$$
\dot{V}_{3}=-z_{1}^{2}-z_{2}^{2}-z_{3}^{2}+z_{3}\left(3 x_{1}+5 x_{2}+3 x_{3}+x_{4}\right)
$$

Now, we define

$$
z_{4}=3 x_{1}+5 x_{2}+3 x_{3}+x_{4}
$$

Using (32), we can simplify the equation (31) as

$$
\dot{V}_{2}=-z_{1}^{2}-z_{2}^{2}-z_{3}^{2}+z_{3} z_{4}
$$

Finally, we define a quadratic Lyapunov function

$$
V\left(z_{1}, z_{2}, z_{3}, z_{4}, e_{a}, e_{b}, e_{c}\right)=V_{3}\left(z_{1}, z_{2}, z_{3}\right)+\frac{1}{2} z_{4}^{2}+\frac{1}{2} e_{a}^{2}+\frac{1}{2} e_{b}^{2}+\frac{1}{2} e_{c}^{2}
$$

which is a positive definite function on $\Re^{7}$.

Differentiating $V$ along the dynamics (15), we get

$$
\dot{V}=-z_{1}^{2}-z_{2}^{2}-z_{3}^{2}-z_{4}^{2}+z_{4}\left(z_{4}+z_{3}+\dot{z}_{4}\right)-e_{a} \dot{\hat{a}}-e_{b} \dot{\hat{b}}-e_{c} \dot{\hat{c}}
$$

Eq. (35) can be written compactly as

$$
\dot{V}=-z_{1}^{2}-z_{2}^{2}-z_{3}^{2}-z_{4}^{2}+z_{4} S-e_{a} \dot{\hat{a}}-e_{b} \dot{\hat{b}}-e_{c} \dot{\hat{c}}
$$

where

$$
S=z_{4}+z_{3}+\dot{z}_{4}=z_{4}+z_{3}+3 \dot{x}_{1}+5 \dot{x}_{2}+3 \dot{x}_{3}+\dot{x}_{4}
$$


A simple calculation gives

$$
S=4 x_{1}+9 x_{2}+(9-a) x_{3}+4 x_{4}-b \sinh \left(x_{2}\right)-c x_{1}^{4} x_{4}+u
$$

Substituting the adaptive control law (18) into (38), we obtain

$$
S=-[a-\hat{a}(t)] x_{3}-[b-\hat{b}(t)] \sinh \left(x_{2}\right)-[c-\hat{c}(t)] x_{1}^{4} x_{4}-k z_{4}
$$

Using the definitions (17), we can simplify (39) as

$$
S=-e_{a} x_{3}-e_{b} \sinh \left(x_{2}\right)-e_{c} x_{1}^{4} x_{4}-k z_{4}
$$

Substituting the value of $S$ from (40) into (36), we obtain

$$
\left\{\begin{aligned}
\dot{V}= & -z_{1}^{2}-z_{2}^{2}-z_{3}^{2}-(1+k) z_{4}^{2}+e_{a}\left(-x_{3} z_{4}-\dot{\hat{a}}\right) \\
& +e_{b}\left(-\sinh \left(x_{2}\right) z_{4}-\dot{\hat{b}}\right)+e_{c}\left(-x_{1}^{4} x_{4} z_{4}-\dot{\hat{c}}\right)
\end{aligned}\right.
$$

Substituting the update law (20) into (41), we get

$$
\dot{V}=-z_{1}^{2}-z_{2}^{2}-z_{3}^{2}-(1+k) z_{4}^{2}
$$

which is a negative semi-definite function on $\Re^{7}$.

From (42), it follows that the vector $z(t)=\left(z_{1}(t), z_{2}(t), z_{3}(t), z_{4}(t)\right)$ and the parameter estimation error $\left(e_{a}(t), e_{b}(t), e_{c}(t)\right)$ are globally bounded, i.e.

$$
\left[\begin{array}{lllllll}
z_{1}(t) & z_{2}(t) & z_{3}(t) & z_{4}(t) & e_{a}(t) & e_{b}(t) & e_{c}(t)
\end{array}\right] \in \boldsymbol{L}_{\infty}
$$

Also, it follows from (42) that

$$
\dot{V} \leqslant-z_{1}^{2}-z_{2}^{2}-z_{3}^{2}-z_{4}^{2}=-\|\mathbf{z}\|^{2}
$$

That is,

$$
\|\mathbf{z}\|^{2} \leqslant-\dot{V}
$$

Integrating the inequality (45) from 0 to $t$, we get

$$
\int_{0}^{t}|z(\tau)|^{2} d \tau \leqslant V(0)-V(t)
$$

From (46), it follows that $z(t) \in \boldsymbol{L}_{2}$.

From Eq. (15), it can be deduced that $\dot{\boldsymbol{z}}(t) \in \boldsymbol{L}_{\infty}$.

Thus, using Barbalat's lemma [90], we conclude that $z(t) \rightarrow \mathbf{0}$ exponentially as $t \rightarrow \infty$ for all initial conditions $\boldsymbol{z}(0) \in \mathfrak{R}^{4}$. 
Hence, it is immediate that $\boldsymbol{x}(t) \rightarrow \mathbf{0}$ exponentially as $t \rightarrow \infty$ for all initial conditions $\boldsymbol{x}(0) \in \mathfrak{R}^{4}$.

This completes the proof.

For the numerical simulations, the classical fourth-order Runge-Kutta method with step size $h=10^{-8}$ is used to solve the system of differential equations (15) and (20), when the adaptive control law (18) is applied.

The parameter values of the novel hyperjerk system (15) are taken as in the hyperchaotic case, viz. $a=3.7, b=0.05, c=1.3$, and the positive gain constant as $k=10$.

Furthermore, as initial conditions of the novel hyperjerk system (15), we take

$$
x_{1}(0)=2.3, x_{2}(0)=-8.5, x_{3}(0)=6.2, x_{4}(0)=3.6
$$

Also, as initial conditions of the parameter estimates, we take

$$
\hat{a}(0)=5.3, \hat{b}(0)=12.4, \hat{c}(0)=7.5
$$

In Fig. 5, the exponential convergence of the controlled states $\left(x_{1}, x_{2}, x_{3}, x_{4}\right)$ is depicted, when the adaptive control law (18) and parameter update law (20) are implemented.

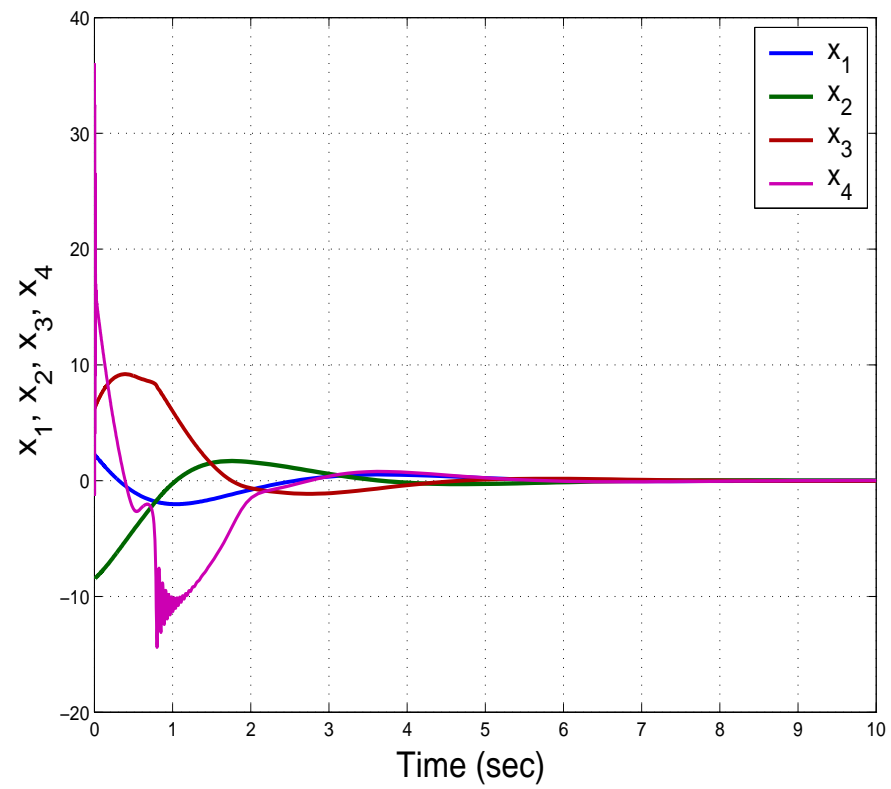

Figure 5: Time-history of the controlled states $x_{1}(t), x_{2}(t), x_{3}(t), x_{4}(t)$ 


\section{Adaptive synchronization of the identical 4-D novel hyperjerk systems with unknown parameters}

In this section, we use backstepping control method to derive an adaptive control law for globally and exponentially synchronizing the identical 4-D novel hyperjerk systems with unknown parameters.

As the master system, we consider the 4-D novel hyperjerk system given by

$$
\left\{\begin{array}{l}
\dot{x}_{1}=x_{2} \\
\dot{x}_{2}=x_{3} \\
\dot{x}_{3}=x_{4} \\
\dot{x}_{4}=-x_{1}-x_{2}-a x_{3}-b \sinh \left(x_{2}\right)-c x_{1}^{4} x_{4}
\end{array}\right.
$$

where $x_{1}, x_{2}, x_{3}, x_{4}$ are the states of the system, and $a, b, c$ are unknown constant parameters.

As the slave system, we consider the 4-D novel hyperjerk system given by

$$
\left\{\begin{array}{l}
\dot{y}_{1}=y_{2} \\
\dot{y}_{2}=y_{3} \\
\dot{y}_{3}=y_{4} \\
\dot{y}_{4}=-y_{1}-y_{2}-a y_{3}-b \sinh \left(y_{2}\right)-c y_{1}^{4} y_{4}+u
\end{array}\right.
$$

where $y_{1}, y_{2}, y_{3}, y_{4}$ are the states of the system, and $u$ is a backstepping control to be determined using estimates $\hat{a}(t), \hat{b}(t)$ and $\hat{c}(t)$ for $a, b$ and $c$, respectively.

We define the synchronization errors between the states of the master system (49) and the slave system (50) as

$$
\left\{\begin{array}{l}
e_{1}=y_{1}-x_{1} \\
e_{2}=y_{2}-x_{2} \\
e_{3}=y_{3}-x_{3} \\
e_{4}=y_{4}-x_{4}
\end{array}\right.
$$

Then the error dynamics is easily obtained as

$$
\left\{\begin{array}{l}
\dot{e}_{1}=e_{2} \\
\dot{e}_{2}=e_{3} \\
\dot{e}_{3}=e_{4} \\
\dot{e}_{4}=-e_{1}-e_{2}-a e_{3}-b\left[\sinh \left(y_{2}\right)-\sinh \left(x_{2}\right)\right]-c\left(y_{1}^{4} y_{4}-x_{1}^{4} x_{4}\right)+u
\end{array}\right.
$$


The parameter estimation errors are defined as:

$$
\left\{\begin{array}{l}
e_{a}(t)=a-\hat{a}(t) \\
e_{b}(t)=b-\hat{b}(t) \\
e_{c}(t)=c-\hat{c}(t)
\end{array}\right.
$$

Differentiating (53) with respect to $t$, we obtain the following equations:

$$
\left\{\begin{array}{l}
\dot{e}_{a}(t)=-\dot{\hat{a}}(t) \\
\dot{e}_{b}(t)=-\dot{\hat{b}}(t) \\
\dot{e}_{c}(t)=-\dot{\hat{c}}(t)
\end{array}\right.
$$

Next, we shall state and prove the main result of this section.

Theorem 2 The identical 4-D novel hyperjerk systems (49) and (50) with unknown parameters $a, b$ and $c$ are globally and exponentially synchronized by the adaptive control law

$$
\left\{\begin{aligned}
u(t)= & -4 e_{1}-9 e_{2}-[9-\hat{a}(t)] e_{3}-4 e_{4}+\hat{b}(t)\left[\sinh \left(y_{2}\right)-\sinh \left(x_{2}\right)\right] \\
& +\hat{c}(t)\left(y_{1}^{4} y_{4}-x_{1}^{4} x_{4}\right)-k z_{4}
\end{aligned}\right.
$$

where $k>0$ is a gain constant,

$$
z_{4}=3 e_{1}+5 e_{2}+3 e_{3}+e_{4}
$$

and the update law for the parameter estimates $\hat{a}(t), \hat{b}(t), \hat{c}(t)$ is given by

$$
\left\{\begin{array}{l}
\dot{\hat{a}}(t)=-e_{3} z_{4} \\
\dot{\hat{b}}(t)=-\left[\sinh \left(y_{2}\right)-\sinh \left(x_{2}\right)\right] z_{4} \\
\dot{\hat{c}}(t)=-\left(y_{1}^{4} y_{4}-x_{1}^{4} x_{4}\right) z_{4}
\end{array}\right.
$$

Proof We prove this result via backstepping control and Lyapunov stability theory [90].

First, we define a quadratic Lyapunov function

$$
V_{1}\left(z_{1}\right)=\frac{1}{2} z_{1}^{2}
$$

where

$$
z_{1}=e_{1}
$$


Differentiating $V_{1}$ along the error dynamics (52), we get

$$
\dot{V}_{1}=z_{1} \dot{z}_{1}=e_{1} e_{2}=-z_{1}^{2}+z_{1}\left(e_{1}+e_{2}\right)
$$

Now, we define

$$
z_{2}=e_{1}+e_{2}
$$

Using (61), we can simplify the equation (60) as

$$
\dot{V}_{1}=-z_{1}^{2}+z_{1} z_{2}
$$

Secondly, we define a quadratic Lyapunov function

$$
V_{2}\left(z_{1}, z_{2}\right)=V_{1}\left(z_{1}\right)+\frac{1}{2} z_{2}^{2}=\frac{1}{2}\left(z_{1}^{2}+z_{2}^{2}\right)
$$

Differentiating $V_{2}$ along the error dynamics (52), we get

$$
\dot{V}_{2}=-z_{1}^{2}-z_{2}^{2}+z_{2}\left(2 e_{1}+2 e_{2}+e_{3}\right)
$$

Now, we define

$$
z_{3}=2 e_{1}+2 e_{2}+e_{3}
$$

Using (65), we can simplify the equation (64) as

$$
\dot{V}_{2}=-z_{1}^{2}-z_{2}^{2}+z_{2} z_{3}
$$

Thirdly, we define a quadratic Lyapunov function

$$
V_{3}\left(z_{1}, z_{2}, x_{3}\right)=V_{2}\left(z_{1}, z_{2}\right)+\frac{1}{2} z_{3}^{2}=\frac{1}{2}\left(z_{1}^{2}+z_{2}^{2}+z_{3}^{2}\right)
$$

Differentiating $V_{3}$ along the error dynamics (52), we get

$$
\dot{V}_{3}=-z_{1}^{2}-z_{2}^{2}-z_{3}^{2}+z_{3}\left(3 e_{1}+5 e_{2}+3 e_{3}+e_{4}\right)
$$

Now, we define

$$
z_{4}=3 e_{1}+5 e_{2}+3 e_{3}+e_{4}
$$

Using (69), we can simplify the equation (68) as

$$
\dot{V}_{2}=-z_{1}^{2}-z_{2}^{2}-z_{3}^{2}+z_{3} z_{4}
$$

Finally, we define a quadratic Lyapunov function

$$
V\left(z_{1}, z_{2}, z_{3}, z_{4}, e_{a}, e_{b}, e_{c}\right)=V_{3}\left(z_{1}, z_{2}, z_{3}\right)+\frac{1}{2} z_{4}^{2}+\frac{1}{2} e_{a}^{2}+\frac{1}{2} e_{b}^{2}+\frac{1}{2} e_{c}^{2}
$$

Clearly, $V$ is a positive definite function on $\Re^{7}$. 
Differentiating $V$ along the error dynamics (52), we get

$$
\dot{V}=-z_{1}^{2}-z_{2}^{2}-z_{3}^{2}-z_{4}^{2}+z_{4}\left(z_{4}+z_{3}+\dot{z}_{4}\right)-e_{a} \dot{\hat{a}}-e_{b} \dot{\hat{b}}-e_{c} \dot{\hat{c}}
$$

Eq. (72) can be written compactly as

$$
\dot{V}=-z_{1}^{2}-z_{2}^{2}-z_{3}^{2}-z_{4}^{2}+z_{4} S-e_{a} \dot{\hat{a}}-e_{b} \dot{\hat{b}}-e_{c} \dot{\hat{c}}
$$

where

$$
S=z_{4}+z_{3}+\dot{z}_{4}=z_{4}+z_{3}+3 \dot{e}_{1}+5 \dot{e}_{2}+3 \dot{e}_{3}+\dot{e}_{4}
$$

A simple calculation gives

$$
S=4 e_{1}+9 e_{2}+(9-a) e_{3}+4 e_{4}-b\left[\sinh \left(y_{2}\right)-\sinh \left(x_{2}\right)\right]-c\left(y_{1}^{4} y_{4}-x_{1}^{4} x_{4}\right)+u
$$

Substituting the adaptive control law (55) into (75), we obtain

$$
S=-[a-\hat{a}(t)] e_{3}-[b-\hat{b}(t)]\left[\sinh \left(y_{2}\right)-\sinh \left(x_{2}\right)\right]-[c-\hat{c}(t)]\left(y_{1}^{4} y_{4}-x_{1}^{4} x_{4}\right]-k z_{4}
$$

Using the definitions (54), we can simplify (76) as

$$
S=-e_{a} e_{3}-e_{b}\left[\sinh \left(y_{2}\right)-\sinh \left(x_{2}\right)\right]-e_{c}\left(y_{1}^{4} y_{4}-x_{1}^{4} x_{4}\right)-k z_{4}
$$

Substituting the value of $S$ from (77) into (73), we obtain

$$
\left\{\begin{aligned}
\dot{V}= & -z_{1}^{2}-z_{2}^{2}-z_{3}^{2}-(1+k) z_{4}^{2}+e_{a}\left(-e_{3} z_{4}-\dot{\hat{a}}\right) \\
& +e_{b}\left[-\left[\sinh \left(y_{2}\right)-\sinh \left(x_{2}\right)\right] z_{4}-\hat{\hat{b}}\right]+e_{c}\left[-\left(y_{1}^{4} y_{4}-x_{1}^{4} x_{4}\right) z_{4}-\dot{\hat{c}}\right]
\end{aligned}\right.
$$

Substituting the update law (57) into (78), we get

$$
\dot{V}=-z_{1}^{2}-z_{2}^{2}-z_{3}^{2}-(1+k) z_{4}^{2},
$$

which is a negative semi-definite function on $\Re^{7}$.

From (79), it follows that the vector $\boldsymbol{z}(t)=\left(z_{1}(t), z_{2}(t), z_{3}(t), z_{4}(t)\right)$ and the parameter estimation error $\left(e_{a}(t), e_{b}(t), e_{c}(t)\right)$ are globally bounded, i.e.

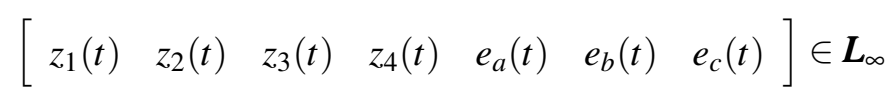

Also, it follows from (79) that

$$
\dot{V} \leqslant-z_{1}^{2}-z_{2}^{2}-z_{3}^{2}-z_{4}^{2}=-\|\mathbf{z}\|^{2}
$$

That is,

$$
\|\mathbf{z}\|^{2} \leqslant-\dot{V}
$$


Integrating the inequality (82) from 0 to $t$, we get

$$
\int_{0}^{t}|z(\tau)|^{2} d \tau \leqslant V(0)-V(t)
$$

From (83), it follows that $z(t) \in \boldsymbol{L}_{2}$.

From Eq. (52), it can be deduced that $\dot{z}(t) \in \boldsymbol{L}_{\infty}$.

Thus, using Barbalat's lemma [90], we conclude that $\boldsymbol{z}(t) \rightarrow \mathbf{0}$ exponentially as $t \rightarrow \infty$ for all initial conditions $z(0) \in \mathfrak{R}^{4}$.

Hence, it is immediate that $\boldsymbol{e}(t) \rightarrow \mathbf{0}$ exponentially as $t \rightarrow \infty$ for all initial conditions $\boldsymbol{e}(0) \in \mathfrak{R}^{4}$.

This completes the proof.

For the numerical simulations, the classical fourth-order Runge-Kutta method with step size $h=10^{-8}$ is used to solve the system of differential equations (49) and (50).

The parameter values of the novel hyperjerk systems are taken as in the hyperchaotic case, viz. $a=3.7, b=0.2, c=1.5$ and the positive gain constant as $k=10$.

Also, as initial conditions of the master system (49), we take

$$
x_{1}(0)=1.5, x_{2}(0)=0.4, x_{3}(0)=-0.8, x_{4}(0)=1.5
$$

As initial conditions of the slave system (50), we take

$$
y_{1}(0)=3.7, y_{2}(0)=1.2, y_{3}(0)=1.6, y_{4}(0)=2.3
$$

Furthermore, as initial conditions of the parameter estimates $\hat{a}(t), \hat{b}(t)$ and $\hat{c}(t)$, we take

$$
\hat{a}(0)=6.1, \hat{b}(0)=3.8, \hat{c}(0)=2.9
$$

In Figs. 6-9, the complete synchronization of the identical 4-D novel hyperchaotic hyperjerk systems (49) and (50) is shown, when the adaptive control law and the parameter update law are implemented.

Also, in Fig. 10, the time-history of the synchronization errors $\left(e_{1}(t), e_{2}(t), e_{3}(t), e_{4}(t)\right)$ is shown.

\section{Conclusion}

In this research work, a 4-D novel hyperchaotic hyperjerk system with two nonlinearities has been proposed, and its qualitative properties have been detailed. The novel hyperjerk system has a unique equilibrium at the origin, which is a saddle-focus and unstable. The Lyapunov exponents of the novel hyperjerk system were obtained as $L_{1}=0.14219, L_{2}=0.04605, L_{3}=0$ and $L_{4}=-1.39267$. The Kaplan-Yorke dimension of the novel hyperjerk system was obtained as $D_{K Y}=3.1348$. Next, an adaptive 


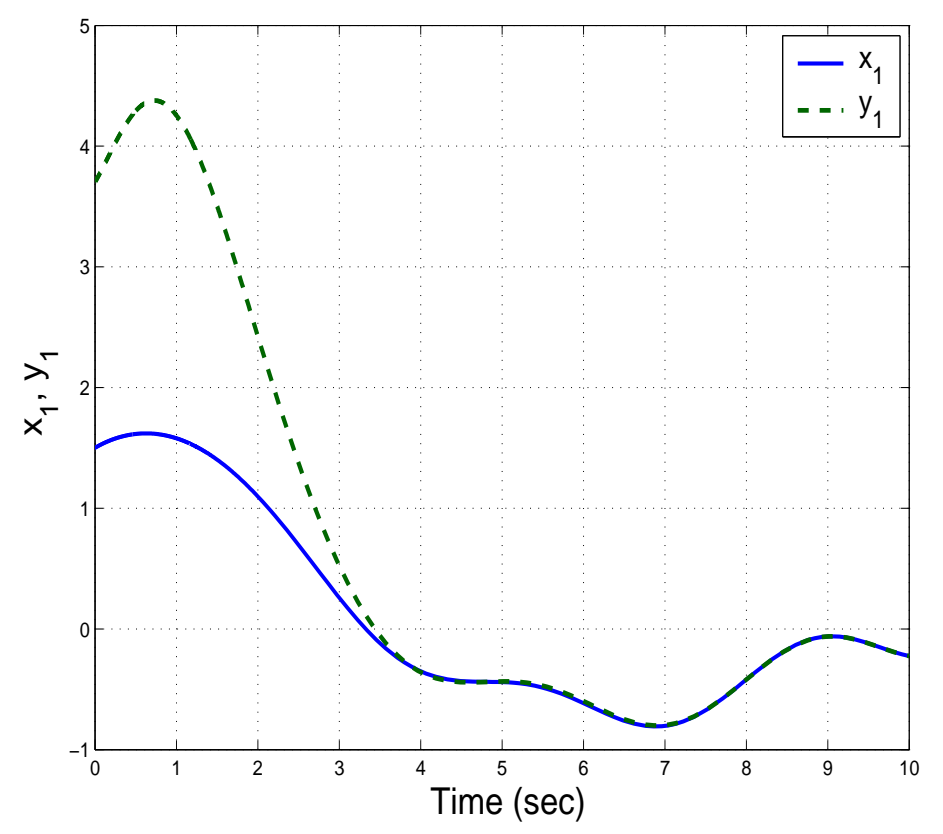

Figure 6: Synchronization of the states $x_{1}(t)$ and $y_{1}(t)$

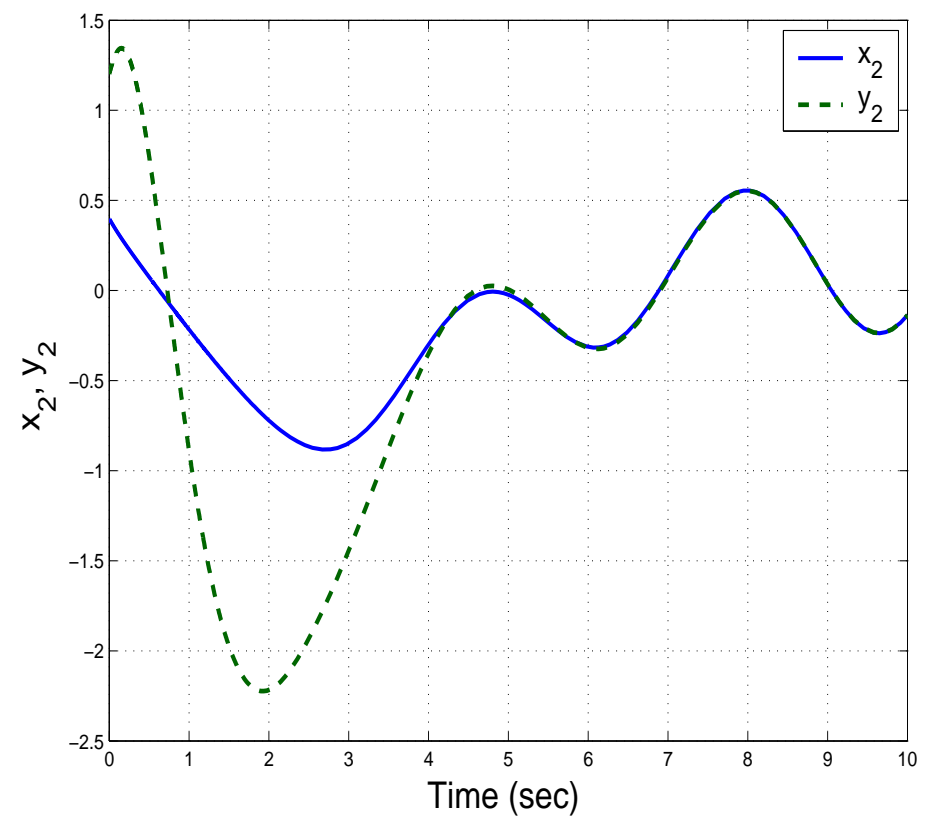

Figure 7: Synchronization of the states $x_{2}(t)$ and $y_{2}(t)$ 


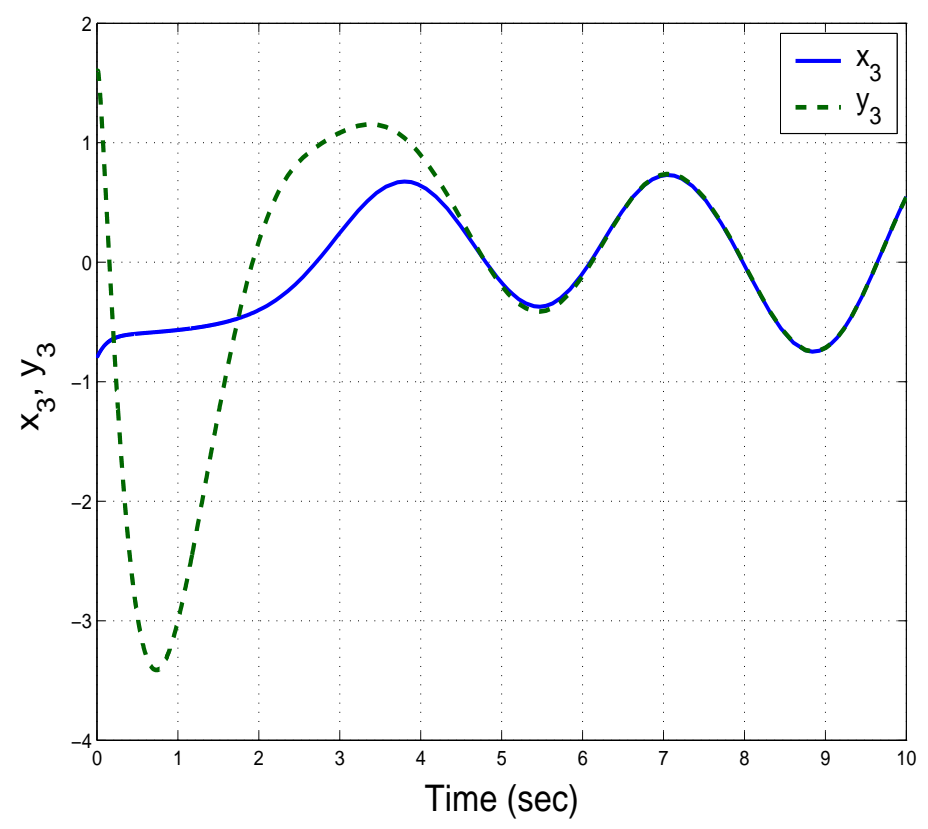

Figure 8: Synchronization of the states $x_{3}(t)$ and $y_{3}(t)$

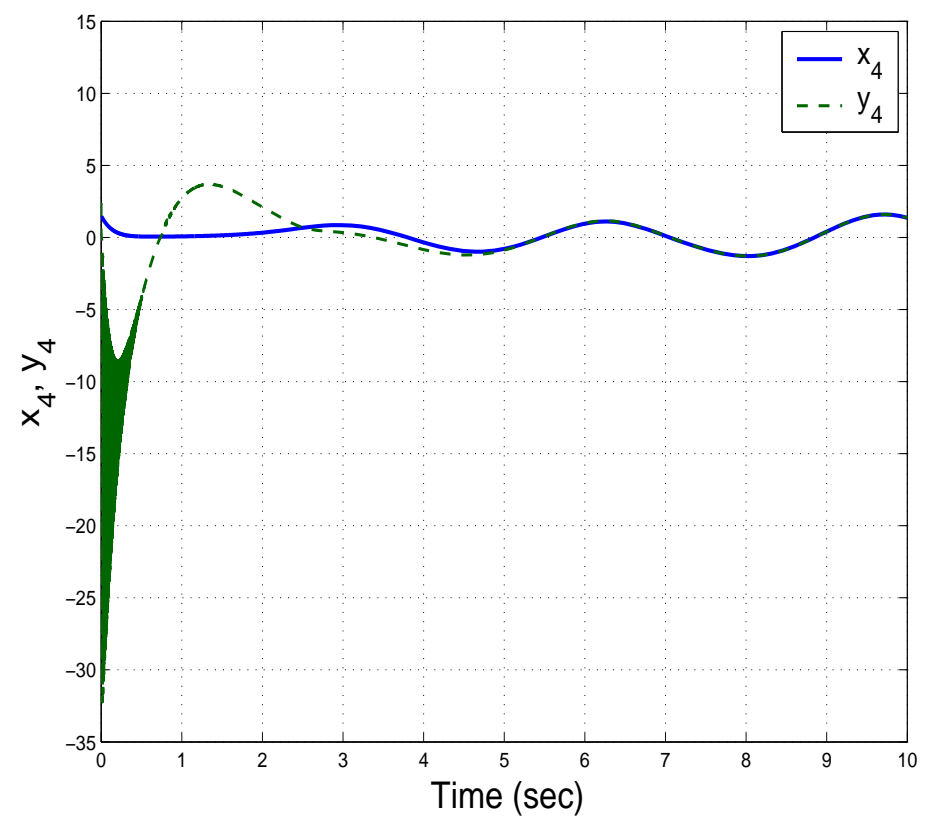

Figure 9: Synchronization of the states $x_{4}(t)$ and $y_{4}(t)$ 


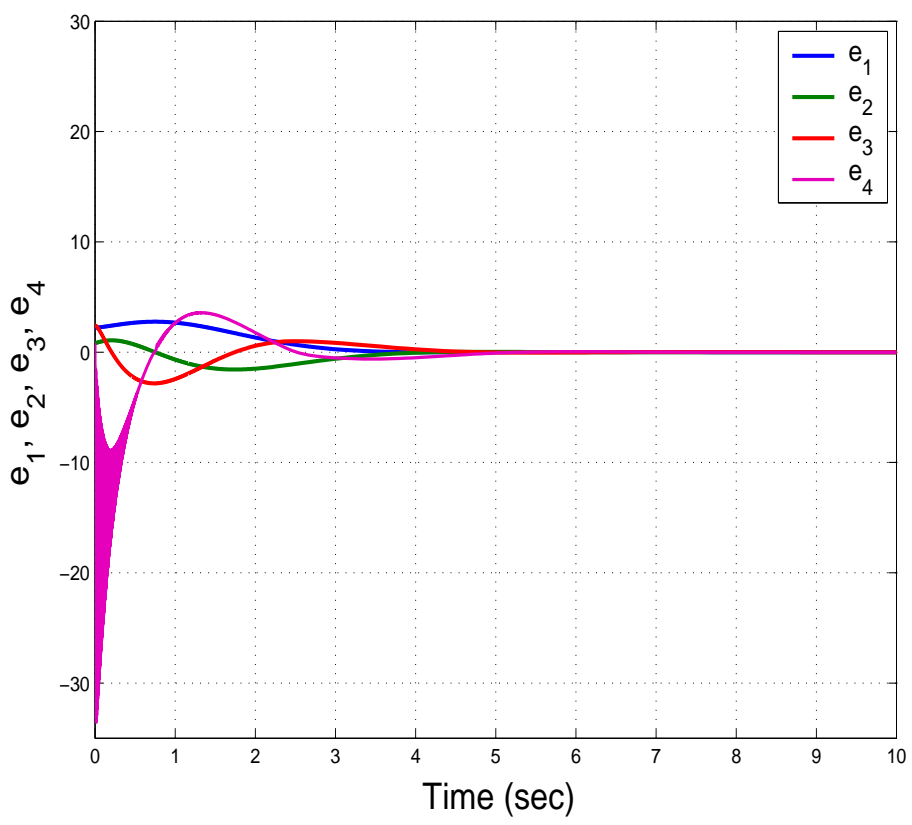

Figure 10: Time-history of the synchronization errors $e_{1}, e_{2}, e_{3}, e_{4}$

controller was designed via backstepping control method to stabilize the novel hyperjerk chaotic system with three unknown parameters. Moreover, an adaptive controller was designed via backstepping control method to achieve global synchronization of the identical novel hyperjerk systems with three unknown parameters. MATLAB simulations have been provided to illustrate all the main results derived in this research work on a novel hyperjerk system.

\section{References}

[1] A.T. AZAR and S. VAIDYANATHAN: Chaos Modeling and Control Systems Design. Berlin, Springer, 2015.

[2] S. VAIDYANATHAN and C.VOLOS: Advances and Applications in Chaotic Systems. Berlin, Springer, 2016.

[3] E.N. LORENZ: Deterministic nonperiodic flow. Journal of the Atmospheric Sciences, 20 (1963), 130-141.

[4] O.E. RÖSSLER: An equation for continuous chaos. Physics Letters A, 57 (1976), 397-398. 
[5] A. ARNEODO, P. COULLET and C. TRESSER: Possible new strange attractors with spiral structure. Communications in Mathematical Physics, 79 (1981), 573579 .

[6] J.C. SPROTT: Some simple chaotic flows. Physical Review E, 50 (1994), 647650.

[7] G. CHEN and T. UETA: Yet another chaotic attractor. International Journal of Bifurcation and Chaos, 9 (1999), 1465-1466.

[8] J. LÜ and G. CHEN: A new chaotic attractor coined. International Journal of Bifurcation and Chaos, 12 (2002), 659-661.

[9] C.X. LIU, T. LIU, L. LIU and K. LIU: A new chaotic attractor. Chaos, Solitons and Fractals, 22 (2004), 1031-1038.

[10] G. CAI and Z. TAN: Chaos synchronization of a new chaotic system via nonlinear control. Journal of Uncertain Systems, 1 (2007), 235-240.

[11] G. TIGAN and D. OPRIS: Analysis of a 3D chaotic system. Chaos, Solitons and Fractals, 36 (2008), 1315-1319.

[12] D. LI: A three-scroll chaotic attractor. Physics Letters A, 372 (2008), 387-393.

[13] V. SUNDARAPANDIAN and I. PEHLIVAN: Analysis, control, synchronization and circuit design of a novel chaotic system. Mathematical and Computer Modelling, 55 (2012), 1904-1915.

[14] V. SUNDARAPANDIAN: Analysis and anti-synchronization of a novel chaotic system via active and adaptive controllers. Journal of Engineering Science and Technology Review, 6 (2013), 45-52.

[15] S. VAIDYANATHAN: A new six-term 3-D chaotic system with an exponential nonlinearity. Far East Journal of Mathematical Sciences, 79 (2013), 135-143.

[16] S. VAIDYANATHAN: Analysis and adaptive synchronization of two novel chaotic systems with hyperbolic sinusoidal and cosinusoidal nonlinearity and unknown parameters. Journal of Engineering Science and Technology Review, 6 (2013), 53-65.

[17] S. VAIDYANATHAN: A new eight-term 3-D polynomial chaotic system with three quadratic nonlinearities. Far East Journal of Mathematical Sciences, 84 (2014), 219-226.

[18] S. VAIDYANATHAN: Analysis, control and synchronisation of a six-term novel chaotic system with three quadratic nonlinearities. International Journal of Modelling, Identification and Control, 22 (2014), 41-53. 
[19] S. VAIDYANATHAN and K. MADHAVAN: Analysis, adaptive control and synchronization of a seven-term novel 3-D chaotic system. International Journal of Control Theory and Applications, 6 (2013), 121-137.

[20] S. VAIDYANATHAN: Analysis and adaptive synchronization of eight-term 3-D polynomial chaotic systems with three quadratic nonlinearities. European Physical Journal: Special Topics, 223 (2014), 1519-1529.

[21] S. VAIDYANATHAN, CH. VOLOS, V.T. PHAM, K. MADHAVAN and B.A. IDOWU: Adaptive backstepping control, synchronization and circuit simulation of a 3-D novel jerk chaotic system with two hyperbolic sinusoidal nonlinearities. Archives of Control Sciences, 24 (2014), 257-285.

[22] S. VAIDYANATHAN: Generalised projective synchronisation of novel 3-D chaotic systems with an exponential non-linearity via active and adaptive control. International Journal of Modelling, Identification and Control, 22 (2014), 207-217.

[23] S. VAIDYANATHAN and C. VOLOS: Analysis and adaptive control of a novel 3-D conservative no-equilibrium chaotic system. Archives of Control Sciences, 25 (3), (2016), 333-353.

[24] S. VAIDYANATHAN: Analysis, properties and control of an eight-term 3-D chaotic system with an exponential nonlinearity. International Journal of Modelling, Identification and Control, 23 (2015), 164-172.

[25] S. VAIDYANATHAN: A 3-D novel highly chaotic system with four quadratic nonlinearities, its adaptive control and anti-synchronization with unknown parameters. Journal of Engineering Science and Technology Review, 8 (2015), 106-115.

[26] S. VAIDYANATHAN: Analysis, control and synchronization of a 3-D novel jerk chaotic system with two quadratic nonlinearities. Kyungpook Mathematical Journal, 55 (2015), 563-586.

[27] S. VAIDYANATHAN: A novel 3-D jerk chaotic system with three quadratic nonlinearities and its adaptive control. Archives of Control Sciences, 26 (1), (2016), $19-47$.

[28] S. VAIDYANATHAN and S. PAKIRISWAMY: A five-term 3-D novel conservative chaotic system and its generalized projective synchronization via adaptive control method. International Journal of Control Theory and Applications, 9 (1), (2016), 61-78.

[29] S. VAIDYANATHAN: A novel 3-D conservative chaotic system with a sinusoidal nonlinearity and its adaptive control. International Journal of Control Theory and Applications, 9 (1), (2016), 115-132. 
[30] S. VAIDYANATHAN: Mathematical analysis, adaptive control and synchronization of a ten-term novel three-scroll chaotic system with four quadratic nonlinearities. International Journal of Control Theory and Applications, 9 (1), (2016), $1-20$.

[31] S. VAIDYANATHAN and K. RAJAGOPAL: Analysis, control, synchronization and LabVIEW implementation of a seven-term novel chaotic system. International Journal of Control Theory and Applications, 9 (1), (2016), 151-174.

[32] S. VAIDYANATHAN and S. PAKIRISWAMY: Adaptive control and synchronization design of a seven-term novel chaotic system with a quartic nonlinearity. International Journal of Control Theory and Applications, 9 (1), (2016), 237-256.

[33] S. VAIDYANATHAN: A highly chaotic system with four quadratic nonlinearities, its analysis, control and synchronization via integral sliding mode control. International Journal of Control Theory and Applications, 9 (1), (2016), 279-297.

[34] S. VAIDYANATHAN: A novel 3-D jerk chaotic system with two quadratic nonlinearities and its adaptive control. International Journal of Control Theory and Applications, 9 (1), (2016), 199-219.

[35] I. PEHLIVAN, I.M. MOROZ and S. VAIDYANATHAN: Analysis, synchronization and circuit design of a novel butterfly attractor. Journal of Sound and Vibration, 333 (2014), 5077-5096.

[36] S. JAFARI and J.C. SPROTT: Simple chaotic flows with a line equilibrium. Chaos, Solitons and Fractals, 57 (2013), 79-84.

[37] V.T. PHAM, C. VOLOS, S. JAFARI, Z. WEI and X. WANG: Constructing a novel no-equilibrium chaotic system. International Journal of Bifurcation and Chaos, 24 (2014), 1450073.

[38] V.T. PHAM, S. JAFARI, S. VAIDYANATHAN, C. VOLOS and X. WANG: A novel memristive neural network with hidden attractors and its circuitry implementation. Science China Technological Sciences, 59 (2016), 358-363.

[39] V.T. PHAM, C. VOLOS, S. JAFARI, S. VAIDYANATHAN, T. KAPITANIAK and X. WANG: A chaotic system with different families of hidden attractors. International Journal of Bifurcation and Chaos, 26 (2016),1650139.

[40] V.T. PHAM, S. VAIDYANATHAN, C.K. VOLOS, S. JAFARI, N.V. KUZNETSOV and T.M. HOANG: A novel memristive time-delay chaotic system without equilibrium points. European Physical Journal: Special Topics, 225 (1), (2016), 127-136. 
[41] S. SAMPATH, S. VAIDYANATHAN, C.K. VOLOS and V.-T. PHAM: An eightterm novel four-scroll chaotic system with cubic nonlinearity and its circuit simulation. Journal of Engineering Science and Technology Review, 8 (2015), 1-6.

[42] A. AKGUL, I. MOROZ, I. PEHLIVAN and S. VAIDYANATHAN: A new fourscroll chaotic attractor and its engineering applications. Optik, 127 (2016), 54915499.

[43] J.C. SPROTT: Elegant Chaos. Singapore, World Scientific, 2010.

[44] C. LI, X. LIAO and K.W. WONG: Lag synchronization of hyperchaos with application to secure communications. Chaos, Solitons and Fractals, 23 (2005), 183193.

[45] N. SMAOUI, A. KAROUMA and M. ZRIBI: Secure communications based on the synchronization of the hyperchaotic Chen and the unified chaotic systems. Communications in Nonlinear Science and Numerical Simulation, 16 (2011), 3279-3293.

[46] T.I. CHIEN and T.L. LIAO: Design of secure digital communication systems using chaotic modulation, cryptography and chaotic synchronization. Chaos, Solitons \& Fractals, 24 (2005), 241-245.

[47] B. ZHANG, M. CHEN and D. ZHOU: Chaotic secure communication based on particle filtering. Chaos, Solitons \& Fractals, 30 (2006), 1273-1280.

[48] Q. ZHANG, L. GUO and X. WEI: A novel image fusion encryption algorithm based on DNA sequence operation and hyper-chaotic system. Optik, 124 (2013), 3596-3600.

[49] H. LIU, X. WANG and A. KADIR: Color image encryption using Choquet fuzzy integral and hyper chaotic system. Optik, 124 (2013), 3257-3533.

[50] A. BUSCARINO, L. FORTUNA and M. FRASCA: Experimental robust synchronization of hyperchaotic circuits. Physica D: Nonlinear Phenomena, 238 (2009), 1917-1922.

[51] N. YUJUN, W. XINGYUAN, W. MINGJUN and Z. HUAGUANG: A new hyperchaotic system and its circuit implementation. Communications in Nonlinear Science and Numerical Simulation, 15 (2010), 3518-3524.

[52] O.E. RÖSSLER: An equation for hyperchaos. Physics Letters A, 71 (1979), 155157.

[53] Q. JIA: Hyperchaos generated from the Lorenz chaotic system and its control. Physics Letters A, 366 (2007), 217-222. 
[54] A. CHEN, J. LU, J. LÜ and S. YU: Generating hyperchaotic Lü attractor via state feedback control. Physica A, 364 (2006), 103-110.

[55] X. LI: Modified projective synchronization of a new hyperchaotic system via nonlinear control. Communications in Theoretical Physics, 52 (2009), 274-278.

[56] J. WANG and Z. CHEN: A novel hyperchaotic system and its complex dynamics. International Journal of Bifurcation and Chaos, 18 (2008), 3309-3324.

[57] D. GHOSH and S. BHATTACHARYA: Projective synchronization of new hyperchaotic system with fully unknown parameters. Nonlinear Dynamics, 61 (2010), $11-21$.

[58] Q. JIA: Hyperchaos generated from the Lorenz chaotic system and its control. Physics Letters A, 366 (2007), 217-222.

[59] S. VAIDYANATHAN: A ten-term novel 4-D hyperchaotic system with three quadratic nonlinearities and its control. International Journal of Control Theory and Applications, 6 (2013), 97-109.

[60] S. VAIDYANATHAN, CH. VOLOS and V.T. PHAM: Hyperchaos, adaptive control and synchronization of a novel 5-D hyperchaotic system with three positive Lyapunov exponents and its SPICE implementation. Archives of Control Sciences, 24 (2014), 409-446.

[61] S. VAIDYANATHAN: Qualitative analysis and control of an eleven-term novel 4-D hyperchaotic system with two quadratic nonlinearities. International Journal of Control Theory and Applications, 7 (2014), 35-47.

[62] S. VAIDYANATHAN and A.T. AZAR: Analysis and control of a 4-D novel hyperchaotic system. Studies in Computational Intelligence, 581 (2015), 3-17.

[63] S. VAIDYANATHAN, CH. K. VOLOS and V.T. PHAM: Hyperchaos, adaptive control and synchronization of a novel 5-D hyperchaotic system with three positive Lyapunov exponents and its SPICE implementation. Archives of Control Sciences, 24 (4), (2014), 409-446.

[64] S. VAIDYANATHAN, C. VOLOS, V.T. PHAM and K. MADHAVAN: Analysis, adaptive control and synchronization of a novel 4-D hyperchaotic hyperjerk system and its SPICE implementation. Archives of Control Sciences, 25 (1), (2015), 135-158.

[65] S. VAIDYANATHAN, C.K. VOLOS and V.T. PHAM: Analysis, control, synchronization and SPICE implementation of a novel 4-D hyperchaotic Rikitake dynamo system without equilibrium. Journal of Engineering Science and Technology Review, 8 (2), (2015), 232-244. 
[66] S. VAIDYANATHAN, V.T. PHAM and C.K. VOLOS: A 5-D hyperchaotic Rikitake dynamo system with hidden attractors. European Physical Journal: Special Topics, 224 (2015), 1575-1592.

[67] S. VAIDYANATHAN and A. BOULKROUNE: A novel hyperchaotic system with two quadratic nonlinearities, its analysis and synchronization via integral sliding mode control. International Journal of Control Theory and Applications, 9 (1), (2016), 321-337.

[68] S. VAIDYANATHAN: An eleven-term novel 4-D hyperchaotic system with three quadratic nonlinearities, analysis, control and synchronization via adaptive control method. International Journal of Control Theory and Applications, 9 (1), (2016), 21-43.

[69] V.T. PHAM, S. VAIDYANATHAN, C. VOLOS, S. JAFARI and S.T. KINGNI: A no-equilibrium hyperchaotic system with a cubic nonlinear term. Optik, 127(6), (2016), 3259-3265.

[70] S. SAMPATH, S. VAIDYANATHAN and V.T. PHAM: A novel 4-D hyperchaotic system with three quadratic nonlinearities, its adaptive control and circuit simulation. International Journal of Control Theory and Applications. 9 (1), (2016), 339-356.

[71] K.E. CHLOUVERAKIS and J.C. SPROTT: Chaotic hyperjerk systems. Chaos, Solitons and Fractals, 28 (2006), 739-746.

[72] G. CAI and W. TU: Adaptive backstepping control of the uncertain unified chaotic system. International Journal of Nonlinear Science, 4 (2007), 17-24.

[73] M.T. YASSEN: Controlling, synchronization and tracking chaotic Liu system using active backstepping design. Physics Letters A, 360 (2007), 582-587.

[74] J.A. LAOYE, U.E. VINCENT and S.O. KAREEM: Chaos control of 4D chaotic systems using recursive backstepping nonlinear controller. Chaos, Solitons and Fractals, 39 (2009), 356-362.

[75] S. VAIDYANATHAN: Adaptive backstepping controller and synchronizer design for Arneodo chaotic system with unknown parameters. International Journal of Computer Science and Information Technology, 4 (2012), 145-159.

[76] S. VAIDYANATHAN and K. RAJAGOPAL: Hybrid synchronization of hyperchaotic Wang-Chen and hyperchaotic lorenz systems by active non-linear control. International Journal of Signal System Control and Engineering Application, 4 (2011), 55-61. 
[77] R. KARTHIKEYAN and V. SUNDARAPANDIAN: Hybrid chaos synchronization of four-scroll systems via active control. Journal of Electrical Engineering, 65 (2014), 97-103.

[78] V. SUNDARAPANDIAN and R. KARTHIKEYAN: Anti-synchronization of Lü and Pan chaotic systems by adaptive nonlinear control. European Journal of Scientific Research, 64 (2011), 94-106.

[79] V. SUNDARAPANDIAN and R. KARTHIKEYAN: Adaptive antisynchronization of Uncertain Tigan and Li Systems. Journal of Engineering and Applied Sciences, 7 (2012), 45-52.

[80] X. TAN, J. ZHANG and Y. YANG: Synchronizing chaotic systems using backstepping design. Chaos, Solitons and Fractals, 16 (2003), 37-45.

[81] S. RASAPPAN and S. VAIDYANATHAN: Global chaos synchronization of WINDMI and Coullet chaotic systems by backstepping control. Far East Journal of Mathematical Sciences, 67 (2012), 265-287.

[82] S. RASAPPAN and S. VAIDYANATHAN: Synchronization of hyperchaotic Liu system via backstepping control with recursive feedback. Communications in Computer and Information Science, 305 (2012), 212-221.

[83] S. RASAPPAN and S. VAIDYANATHAN: Hybrid synchronization of $n$-scroll Chua circuits using adaptive backstepping control design with recursive feedback. Malaysian Journal of Mathematical Sciences, 7 (2013), 219-226.

[84] V. SUNDARAPANDIAN and S. SIVAPERUMAL: Sliding controller design of hybrid synchronization of four-wing chaotic systems. International Journal of Soft Computing, 6 (2011), 224-231.

[85] S. VAIDYANATHAN: Global chaos control of hyperchaotic Liu system via sliding control method. International Journal of Control Theory and Applications, 5 (2012), 117-123.

[86] S. VAIDYANATHAN and S. SAMPATH: Anti-synchronization of four-wing chaotic systems via sliding mode control. International Journal of Automatic Computing, 9 (2012), 274-279.

[87] S. VAIDYANATHAN: Global chaos synchronisation of identical Li-Wu chaotic systems via sliding mode control. International Journal of Modelling, Identification and Control, 22 (2014), 170-177.

[88] S. VAIDYANATHAN and S. SAMPATH: Anti-synchronization of identical chaotic systems via novel sliding control method with application to Vaidyanathan-Madhavan chaotic system. International Journal of Control Theory and Applications, 9 (1), (2016), 85-100. 
[89] S. SAMPATH and S. VAIDYANATHAN: Hybrid synchronization of identical chaotic systems via novel sliding control method with application to Sampath four-scroll chaotic system. International Journal of Control Theory and Applications, 9 (1), (2016), 221-235.

[90] H.K. Khalil: Nonlinear Systems, Prentice Hall, New Jersey, 2002. 